ORIGINAL ARTICLE

\title{
Predictors of multi-organ dysfunction in heatstroke
}

\author{
G M Varghese, G John, K Thomas, O C Abraham, D Mathai
}

Emerg Med J 2005;22:185-187. doi: 10.1136/emj.2003.009365

Background: Heatstroke is a medical emergency that results from failure of thermoregulatory mechanism coupled with an exaggerated acute phase response, causing an elevation in core body temperature that rises above $40^{\circ} \mathrm{C}$, producing multi-organ dysfunction. It carries a high mortality rate, and in survivors, a risk of permanent neurological damage.

Objective: To investigate predictors of multiple organ dysfunction syndrome in patients presenting with heatstroke.

See end of article for authors' affiliations .....................

Correspondence to: G M Varghese, Christian Medical College, Vellore, Department of Medicine Unit 2, Vellore, India, 632004; georgemvarghese@ hotmail.com

Accepted 20 November 2003
Methods: We investigated 28 patients admitted to a hospital in southern India during the period January 1998 to December 2001. Using a standard form, we collected data on the patients' characteristics, laboratory data, and outcome, and compared those with multiple organ dysfunction with those without such dysfunction.

Results: We found that more than three quarters of the studied patients developed multiple organ dysfunction, with the most common dysfunction being respiratory failure. Among the selected predictors, metabolic acidosis 14 of 16 patients, $87.5 \%$; $p=0.011$, elevated CPK 17 of 19 patients, 89.5\%; $p=0.005$, and liver enzymes elevated more than twice the normal ( 11 of 18 patients, $61 \% ; p=0.02$ ) had the highest correlation with dysfunction of two or more organs.

Conclusions: The high mortality observed in heatstroke is secondary to multi-organ dysfunction, and among the various parameters assessed, high levels of CPK (>1000 IU/I), metabolic acidosis, and elevated liver enzymes are predictive. Aggressive measures to lower the body temperature with other supportive therapy could substantially reduce the mortality.
$\mathrm{H}$ eatstroke is a common catastrophic medical emergency causing multi-organ dysfunction, which occurs in high temperatures during the summer months. This life threatening illness results from failure of thermoregulatory mechanism coupled with an exaggerated acute phase response, causing an elevation in core body temperature that rises above $40^{\circ} \mathrm{C}$, producing multi-organ dysfunction. The high mortality rate and permanent neurological damage due to heatstroke demands urgent attention. ${ }^{1}$ Early recognition, initiation of rapid cooling measures, and other supportive therapy can reduce the high fatality rate in such patients.

The reported incidence of heatstroke is imprecise, as it is grossly underdiagnosed, and varying definition of heat related illness exist. At least 240 people die of heat related illnesses in the USA each year. ${ }^{2}$ In 1980, 1700 people died in the USA during a prolonged heatwave, ${ }^{2}$ and a sustained heatwave from 12 to 20 July 1995 in Chicago resulted in more than 600 excess deaths. ${ }^{3}$ The incidence of heatstroke varies from 17.6 to 26.5 cases per 100000 population in the USA. ${ }^{4}$ In France, the August heatwave in 2003 caused the unexpected and unprecedented death of $>10000$ people. ${ }^{5}$ In an observational study from the UK, the mean annual heat related mortality was found to be 304 in North Finland and 445 in Athens. ${ }^{6}$ In comparison, in a country with a desert climate such as Saudi Arabia, the incidence varies seasonally, from 22 to 250 cases per 100000 population. ${ }^{7}$ Proper data from most of the world's hot countries are imprecise as this problem is grossly underdiagnosed and under-reported. The incidence of heat related illness and death is likely to increase with predicted incidence of global warming and increasing frequency of heatwaves. ${ }^{8}$

Heatstroke has been classified as exertional or classic. Exertional heatstroke is precipitated by heavy exertion in extremely hot and humid climates and is usually seen in otherwise healthy young people. ${ }^{9}$ Classic heatstroke results from unabated exposure to high temperatures and humidity.
Most people affected by classic heatstroke are very young or elderly, are poor and socially isolated, have underlying medical illness, and do not have access to air conditioning. ${ }^{10}{ }^{11}$ Frequently encountered complications include acute respiratory distress syndrome, disseminated intravascular coagulation (DIC), shock, rhabdomyolysis, renal failure, cerebral oedema, seizures, and hepatic dysfunction. ${ }^{12}$ Laboratory studies may reveal coagulopathy, azotaemia, elevated liver and muscle enzymes, and leucocytosis. ${ }^{13}$

Multi-organ injury results from a complex interplay between the cytotoxic effect of the heat and the inflammatory and coagulation responses of the host, and this leads to multi-organ dysfunction in heatstroke. ${ }^{14}$ Heatstroke has an inherently high mortality due to multi-organ dysfunction, and those who require admission to intensive care units have substantial increase in mortality. ${ }^{1}$ The objective of this study was to investigate predictors of multiple organ dysfunction syndrome in patients presenting with heatstroke.

\section{PATIENTS AND METHODS}

Adult patients admitted to a 1900 bed teaching hospital in southern India with heatstroke from January 1998 to December 2001 were included in the study. In this retrospective study, patients were identified by review of medical intensive care records and medical records ICD codes. Medical records of all consecutive patients with suspected heatstroke were reviewed. Heatstroke was defined as hyperthermia with a core body temperature of more than $105^{\circ} \mathrm{F}\left(40.6^{\circ} \mathrm{C}\right)$ with associated central nervous system dysfunction, during periods of sustained high ambient temperature. ${ }^{15}$ Diagnostic criteria for inclusion were: $(a)$ core body temperature $>105^{\circ} \mathrm{F}$; $(b)$ evidence of central nervous

Abbreviations: ALT, alanine aminotransferase; AST, aspartate aminotransferase; CPK, creatinine phosphokinase; DIC, disseminated intravascular coagulation; LDH, lactate dehydrogenase 
system dysfunction (seizures or altered senses (disorientation, delirium, coma)) documented by the attending physician; and $(c)$ occurrence during summer months with high ambient temperature. Excluded were those with proven central nervous system infection, systemic sepsis, malaria, neuroleptic malignant syndrome, or malignant hyperthermia secondary to anaesthetic agents.

Relevant data were recorded using a predesigned data collection form. The data included demographic characteristics, known pre-morbid illness, temperature at admission and maximum temperature, Glasgow coma scale, clinical features at presentation, vital parameters, laboratory data, (serum creatinine phosphokinase, lactate dehydrogenase $(\mathrm{LDH})$, serum bicarbonate, leukocyte count, creatinine, liver function test, and chest radiography findings), length of intensive care unit and hospital stay, condition at discharge, and outcome.

Organ system dysfunction was assessed using clinical and laboratory parameters as defined by the criteria presented in table $1 .{ }^{16}$ The association between the final outcome measure of multiple (two or more) organ dysfunction with various initial parameters was evaluated. The parameters evaluated were elevated creatinine phosphokinase (>1000 IU/l), LDH $(>500 \mathrm{U} / \mathrm{l})$, liver enzymes (aspartate aminotransferase/alanine aminotransferase; AST/ALT) more than twice normal $(>80 \mathrm{U} / \mathrm{l})$, presence of metabolic acidosis $\left(\mathrm{HCO}_{3}<18 \mathrm{mEq} / \mathrm{l}\right.$ and $\mathrm{pH}<7.35$ ) and leukocytosis (white blood cell count $>11000 / \mathrm{mm}^{3}$ ).

Nominal data were compared by using $\chi^{2}$ analysis or Fisher's exact test of contingency tables.

\section{RESULTS}

Data of 28 patients who fulfilled the inclusion criteria were analysed. Mean age of the patients was 50.6 years (range 20$85)$ with a slightly higher proportion of women (57\%). The mean temperature at admission was $105.7^{\circ} \mathrm{C}$. Mean duration of fever prior to admission was 4.1 days in the multiple organ dysfunction group and 2.3 days in the group with no multiple organ dysfunction. Pre-morbid medical illnesses were present in 18 patients and the patient characteristics are presented in table 2.

Various organ dysfunctions among these patients are presented in table 3. The commonest organ dysfunction was respiratory failure, occurring in 24 patients $(85.7 \%)$. Of

Table 1 Organ system dysfunction criteria

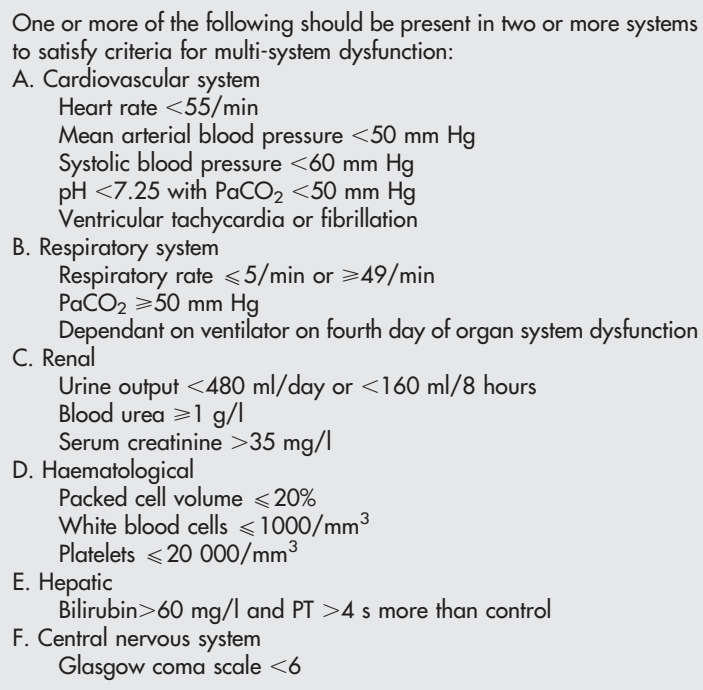

Table 2 Patient characteristics

\begin{tabular}{|c|c|c|}
\hline \multirow[b]{2}{*}{ Characteristic } & \multicolumn{2}{|c|}{ Multi-organ dysfunction } \\
\hline & $\begin{array}{l}\text { Present } \\
(n=22)\end{array}$ & $\begin{array}{l}\text { Absent } \\
(n=6)\end{array}$ \\
\hline Age (mean years) & 48.5 & 60.2 \\
\hline Sex: $M=/ F$ & $8 / 14$ & $4 / 2$ \\
\hline $\begin{array}{l}\text { Mean duration of fever prior to } \\
\text { admission (days) }\end{array}$ & 4.1 & 2.3 \\
\hline \multicolumn{3}{|l|}{ Premorbid illness } \\
\hline Diabetes mellitus & 5 & 4 \\
\hline Hypertension & 6 & 2 \\
\hline Cardiac disease & 2 & 1 \\
\hline Psychiatric illness & 2 & 1 \\
\hline Cerebrovascular accident & 2 & None \\
\hline Temperature at admission $(F)$ & 106.1 & 105.4 \\
\hline Heart rate/min (mean) & 134 & 117 \\
\hline Mean systolic bood pressure $(\mathrm{mm} \mathrm{Hg})$ & 110 & 140 \\
\hline Mortality & 19 & 1 \\
\hline
\end{tabular}

these, $78.6 \%$ required ventilatory support. Elevated creatinine $(>120 \mu \mathrm{mol} / \mathrm{l})$ was present in 18 patients $(64.3 \%)$. The mean value of creatinine phosphokinase (CPK) was $3108 \mathrm{U} / \mathrm{l}$. Of the 25 patients who had a CPK estimation performed, 24 (96\%) had elevated levels $>200 \mathrm{U} / \mathrm{l}$. Eleven $(61 \%)$ of 18 patients who had liver enzyme (AST/ALT) estimated had values exceeding twice the normal levels. Leukocytosis $\left(>11000 / \mathrm{mm}^{3}\right)$ was seen in 16 of 24 patients $(66.7 \%)$ and thrombocytopenia in 8 of 15 patients tested $(53 \%)$.

The overall case fatality rate was $71.4 \%$. Dysfunction of two or more organs was seen in 22 patients $(78.6 \%)$, with a mortality rate of $86.4 \%$. A significant decline in case fatality was seen from 1998 ( 13 deaths in 14 patients) $(92.8 \%)$ to 2001 (four deaths in eight patients) $(50 \%)(p=0.01)$.

Among the selected predictors, metabolic acidosis (14 of 16 patients, $87.5 \% ; p=0.011)$, elevated CPK of $>1000$ IU/1 (17 of 19 patients, $89.5 \% ; \mathrm{p}=0.005$ ) and elevated liver enzymes more than twice the normal (11 of 13 patients, 84.6\%; $\mathrm{p}=0.02$ ) had the highest correlation with dysfunction of two or more organs (table 4). Leucocytosis or high LDH had no statistically significant association with multi-organ dysfunction.

\section{DISCUSSION}

This retrospective study was performed to identify predictors of development of multiple organ dysfunction among patients with heatstroke requiring hospitalisation during the period January 1998 to December 2001. We found that more than three quarters of the studied patients developed multiple organ dysfunction. The overall case fatality rate was more than $70 \%$ and the mortality was even higher $(85 \%)$ in patients with dysfunction of two or more organs. With increased awareness of this problem among healthcare workers, a significant decline in case fatality rate has occurred. Elevated CPK, transaminases, and metabolic acidosis at admission predict development of multiple organ dysfunction and hence poor outcome.

\begin{tabular}{|c|c|c|}
\hline Organ system & $\mathbf{n}$ & $\%$ \\
\hline Respiratory & 24 & 85.7 \\
\hline Central nervous system & 17 & 60.7 \\
\hline Cardiovascular & 15 & 53.6 \\
\hline Renal & 14 & 50.0 \\
\hline
\end{tabular}


Table 4 Predictors of multiorgan dysfunction at admission

\begin{tabular}{llll}
\hline & \multicolumn{2}{l}{ Multi-organ dysfunction } & \\
\cline { 2 - 3 } & $\begin{array}{l}\text { Present } \\
(\mathbf{n}=\mathbf{2 2})\end{array}$ & $\begin{array}{l}\text { Absent } \\
(\mathbf{n}=6)\end{array}$ & $\mathbf{p}$ \\
\hline CPK (>1000 U/I) & $17(n=19)$ & $2(n=6)$ & 0.005 \\
Metabolic acidosis & $14(n=16)$ & $1(n=5)$ & 0.011 \\
Elevated liver enzymes & $11(n=13)$ & $1(n=5)$ & 0.022 \\
(AST/ALT >twice normal) & & & \\
Elevated LDH (>500 U/l) & $7(n=8)$ & $2(n=3)$ & 0.425 \\
Leukocytosis (WBC & $13(n=18)$ & $3(n=6)$ & 0.317 \\
$\left.>11000 / \mathrm{mm}^{3}\right)$ & & & \\
\hline
\end{tabular}

CPK, creatinine phosphokinase; AST, aspartate aminotransferase; ALT, alanine aminotransferase; $L D H$, lactate dehydrogenase; WBC, white blood cells.

The higher mortality in this study compared with previous reports $^{2}{ }^{17}$ could be due to multiple factors. Many of these patients presented late; the mean duration of fever at presentation was 4.1 days among patients with multi-organ dysfunction compared with 2.3 days among those without dysfunction. Because this study was based in a tertiary care hospital, only severe disease may be represented. Awareness of the problem of multi-organ dysfunction was lower among these healthcare workers and therefore aggressive cooling measures were not instituted initially. Such delay, caused by instigating other investigations such as CT scan and lumbar puncture to rule out other causes, could have contributed to the higher mortality.

The most common organ dysfunction was respiratory failure $(85.7 \%)$, with more than three quarters requiring mechanical ventilation as shown in previous reports. Hypotension and tachyarrhythmia has been well documented. ${ }^{18} 19$ Renal dysfunction in heatstroke could be multifactorial. Pre-renal insult, direct heat related injury to the kidney, rhabdomyolysis, and DIC could contribute to renal dysfunction in heatstroke. ${ }^{20}{ }^{21}$ In our patients, a very high level of CPK, despite a lack of history of exercise, was seen. Although thrombocytopenia and DIC were present in some patients, they were lacking in most. Elevated liver enzymes have been documented in heatstroke and are believed to be due to direct thermal injury to the liver. ${ }^{22}{ }^{23}$

Because this study was a retrospective analysis, there are some limitations. There was inadequate documentation. Variation in diagnostic investigations and changes in practices could have influenced the outcome. In addition, as the number of patients was small, we could not perform multiple logistic regression. A prospective study could reduce the potential for such biases, though it might be difficult to obtain a sufficiently large sample.

In conclusion, the high mortality observed in heatstroke is secondary to multi-organ dysfunction, and among the various parameters assessed, high levels of CPK (>1000 IU/l), metabolic acidosis, and elevated liver enzymes were found to be associated with dysfunction of two or more organs. Increasing awareness of this problem among healthcare workers, early recognition, and aggressive measures to lower the body temperature with other supportive therapy could substantially reduce mortality. Establishing a scoring system for stratification of severity and prognostication with these and other parameters is now necessary for the optimal care of this under-recognised but common problem in hot countries.

\section{Authors' affiliations \\ G M Varghese, G John, K Thomas, O C Abraham, D Mathai}

Competing interests: none declared

\section{REFERENCES}

1 Dematte JE, O'Mara K, Buescher J, et al. Near-fatal heat stroke during the 1995 heat wave in Chicago. Ann Intern Med 1998;129:173-81.

2 Centers for Disease Control. Heat-related illness and deaths-United States, 1994-1995. MMWR Morb Mortal Wkly Rep 1995:44:465-8.

3 Centers for Disease Control. Heat-related mortality-Chicago, July 1995. MMWR Morb Mortal Wkly Rep 1995;44:577-9.

4 Jones TS, Liang AP, Kilbourne EM, et al. Morbidity and mortality associated with the July 1980 heat wave in St. Louis and Kansas City, MO. JAMA 1982;247:3327-31.

5 Dorozynski A. Chirac announces investigation into heat wave's death toll. BMJ 2003;327:465.

6 Keating WR, Donaldson GC, Cordioli E, et al. Heat related mortality in warm and cold regions of Europe: observational study. BMJ 2000;321:670-3.

7 Ghaznawi HI, Ibrahim MA. Heat stroke and heat exhaustion in pilgrims performing the Hai (annual pilgrimage) in Saudi Arabia. Ann Saudi Med 1987;7:323-6

8 Katsouyanni K, Trichopoulos D, Zavitsanos X, et al. The 1987 Athens heatwave. Lancet 1988;2:573.

9 Simon HB. Hyperthermia. N Engl J Med 1993;329:483-7.

10 Semenza JC, Rubin $\mathrm{CH}$, Falter $\mathrm{KH}$, et al. Heat-related deaths during the July 1995 heat wave in Chicago. N Engl J Med 1996;335:84-90.

11 Kilbourne EM, Choi K, Jones TS, et al. Risk factors for heatstroke: a casecontrol study. JAMA 1982;247:3332-6.

12 Tek D, Olshaker JS. Heat illness. Emerg Med Clin North Am 1992;10:299-310.

13 Khosla R, Guntupalli KK. Heat-related illnesses. Crit Care Clin 1999:15:251-63.

14 Bouchama A, Knochel JP. Heat stroke. N Eng J Med 2002;346:1978-88.

15 Bross MH, Nash BT, Carlton FB. Heat emergencies. Am Fam Physician 1994; 50:389-96.

16 Knaus WA, Wagner DP. Multiple systems organ failure: epidemiology and prognosis. Crit Care Clin 1989:5:221-32.

17 Clowes GH Jr, O'Donnell TF Jr. eat stroke. N Engl J Med 1974;291:564-7.

18 Shibolet S, Coll R, Gilant T, et al. Heatstroke: its clinical picture and mechanism in 36 cases. Q J Med 1967;36:525-48.

19 Rowell LB, Brengelmann GR, Blackmon JR, et al. Redistribution of blood flow during sustained high skin temperature in resting man. J Appl Physiol 1970;28:415-20

20 Raju SF, Robinson GH, Bower JO. The pathogenesis of acute renal failure in heat stroke. South Med J 1973;66:330-3.

21 Kew MC, Abrahams C, Levin NW, et al. The effects of heatstroke on the function and structure of the kidney. Q J Med 1967;36:277-300.

22 Kew M, Bersohn I, Seftel H, et al. Liver damage in heatstroke. Am J Med 1970;49: 192-202.

23 Hassanein T, Razack A, Gavaler JS, et al. Heatstroke: its clinical and pathological presentation, with particular attention to the liver. Am J Gastroenterol 1992;87:1382-9. 